\title{
X-RAY VARIABILITY DURING THE QUIESCENT STATE OF THE NEUTRON STAR X-RAY TRANSIENT IN THE GLOBULAR CLUSTER NGC 6440
}

\section{Citation}

Cackett, Edward M., Rudy Wijnands, Craig O. Heinke, Peter D. Edmonds, Walter H. G. Lewin, David Pooley, Jonathan E. Grindlay, Peter G. Jonker, and Jon M. Miller. 2005. “X\#Ray Variability during the Quiescent State of the Neutron Star X\#Ray Transient in the Globular Cluster NGC 6440." The Astrophysical Journal 620 (2): 922-28. https://doi.org/10.1086/427178.

\section{Permanent link}

http://nrs.harvard.edu/urn-3:HUL.InstRepos:41399931

\section{Terms of Use}

This article was downloaded from Harvard University's DASH repository, and is made available under the terms and conditions applicable to Other Posted Material, as set forth at http:// nrs.harvard.edu/urn-3:HUL.InstRepos:dash.current.terms-of-use\#LAA

\section{Share Your Story}

The Harvard community has made this article openly available.

Please share how this access benefits you. Submit a story.

\section{Accessibility}




\title{
X-RAY VARIABILITY DURING THE QUIESCENT STATE OF THE NEUTRON STAR X-RAY TRANSIENT IN THE GLOBULAR CLUSTER NGC 6440
}

\author{
Edward M. Cackett, ${ }^{1}$ Rudy Wijnands, ${ }^{2}$ Craig O. Heinke, ${ }^{3}$ Peter D. Edmonds, ${ }^{3}$ Walter H. G. Lewin, ${ }^{4}$ \\ David Pooley, ${ }^{4}$ Jonathan E. Grindlay, ${ }^{3}$ Peter G. Jonker, ${ }^{3}$ and Jon M. Miller ${ }^{3}$ \\ Received 2004 July 20; accepted 2004 November 1
}

\begin{abstract}
The globular cluster NGC 6440 is known to harbor a bright neutron star X-ray transient. We observed the globular cluster with Chandra on two occasions when the bright transient was in its quiescent state, in 2000 July and 2003 June (both observations were made nearly $2 \mathrm{yr}$ after the end of their preceding outbursts). The quiescent spectrum during the first observation is well represented by a two-component model (a neutron star atmosphere model plus a power-law component that dominates at energies above $2 \mathrm{keV}$ ). During the second observation (which was roughly of equal duration to the first observation) we found that the power-law component could no longer be detected. Our spectral fits indicate that the effective temperature of the neutron star surface was consistent between the two observations. We conclude that the effect of the change in the power-law component caused the $0.5-10 \mathrm{keV}$ flux to be a factor of $\sim 2$ lower during the second observation compared to the first observation. We discuss plausible explanations for the variations, including variable residual accretion onto the neutron star magnetosphere or some variation in the interaction of the pulsar wind with the matter still outflowing from the companion star.
\end{abstract}

Subject headings: globular clusters: individual (NGC 6440) — stars: neutron — X-rays: binaries

Online material: color figures

\section{INTRODUCTION}

$\mathrm{X}$-ray transients are a subgroup of low-mass X-ray binariesbinary systems in which a compact object (either a neutron star or a black hole) is accreting matter from a low-mass companion star. They are normally in a quiescent state, during which very little or no accretion onto the compact object occurs. Occasionally, however, these systems go into outburst, lasting weeks to months, throughout which they increase greatly in brightness. Such outbursts arise because of a large increase in the mass accretion rate onto the compact object.

About a dozen neutron star X-ray transient systems have been detected in quiescence as well as in outburst. The quiescent luminosity of these neutron star systems is typically $10^{32}$ $10^{34} \mathrm{ergs} \mathrm{s}^{-1}$, compared to typical outburst luminosities in the range $10^{36}-10^{38} \mathrm{ergs} \mathrm{s}^{-1}$. The X-ray spectra of these quiescent neutron star systems are generally characterized by two components: a soft component (which dominates the spectra below a few $\mathrm{keV}$ ), equivalent to a blackbody temperature of $k T \sim$ $0.1-0.3 \mathrm{keV}$, and a hard power-law X-ray tail (which dominates the spectra above a few $\mathrm{keV}$ ). To explain the X-ray emission of neutron star X-ray transients in their quiescent state, several models have been proposed (for example, residual accretion onto the neutron stars, e.g., Menou et al. 1999; Menou \& McClintock 2001; Campana \& Stella 2000), but the most widely used model is that in which the emission is due to the cooling of the neutron star, which has been heated during the outbursts (see, e.g., Brown et al. 1998). In the Brown et al.

\footnotetext{
${ }^{1}$ School of Physics and Astronomy, University of St. Andrews, North Haugh, St. Andrews, Fife, KY16 933, Scotland, UK; emc14@st-and.ac.uk.

2 Astronomical Institute "Anton Pannekoek," University of Amsterdam, Kruislaan 403, 1098 SJ, Amsterdam, Netherlands.

3 Harvard-Smithsonian Center for Astrophysics, 60 Garden Street, Cambridge, MA 02138 .

${ }^{4}$ Center for Space Research, Massachusetts Institute of Technology, 77 Massachusetts Avenue, Cambridge, MA 02139.
}

(1998) model, the neutron star core is heated by nuclear reactions occurring deep in the crust as a result of accretion during outburst, and this heat is released as thermal emission during quiescence. The X-ray emission from the cooling of a neutron star cannot directly account for the hard power-law tail seen in the X-ray spectra, and the nature of this hard component is uncertain. Possible explanations for the power-law tail are accretion onto the neutron star magnetosphere or pulsar shock emission (e.g., see the discussion in Campana et al. 1998a).

Globular clusters are ideal for studying X-ray transients in quiescence, as the distance to the host clusters can usually be determined more accurately than for the Galactic quiescent $\mathrm{X}$-ray binaries. Here we report on Chandra observations of the globular cluster NGC 6440, which is known to harbor a bright neutron star X-ray transient. NGC 6440 is a globular cluster at a distance of $8.5 \pm 0.4 \mathrm{kpc}$ and reddened by $E(B-V)=1.0$ (Ortolani et al. 1994). A transient in NGC 6440 was first seen during an outburst in 1971 December to 1972 January (Markert et al. 1975; Forman et al. 1976). In 1998 August, a second outburst was detected by the Wide Field Camera (WFC) on board BeppoSAX (in 't Zand et al. 1998) and the All-Sky Monitor (ASM) on board the Rossi X-ray Timing Explorer (RXTE). During this outburst, four type I X-ray bursts were detected (in 't Zand et al. 1999), demonstrating the neutron star nature of the compact object. An optical follow-up of this outburst found two possible candidates, V1 and V2, for the optical counterpart of the transient (Verbunt et al. 2000).

In 't Zand et al. (2001) and Pooley et al. (2002) reported on a Chandra observation of NGC 6440 nearly 2 yr after the end of the 1998 outburst. The neutron star X-ray transient was in quiescence, and Pooley et al. (2002) detected 24 lowluminosity X-ray sources in this globular cluster. They also reported that 4-5 quiescent neutron stars might be present in this cluster, based on their X-ray luminosities and soft, thermal X-ray spectra. Their source CX1 was the brightest lowluminosity source in the cluster, and its position was consistent 
with that of V1 in Verbunt et al. (2000) and possibly the quiescent counterpart of the bright transient source. This issue was resolved in 2001 August, when the RXTE ASM and the BeppoSAX WFC detected another outburst from the transient in NGC 6440. In 't Zand et al. (2001) obtained a brief Chandra observation during this outburst, which resulted in a subarcsecond position of the source. They found that the 1998 and 2001 transient is associated with CX1 from Pooley et al. (2002) and V1 from Verbunt et al. (2000). From here on, we refer to the quiescent counterpart of the neutron star transient in NGC 6440 as CX1 after Pooley et al. (2002).

To study the quiescent counterpart CX1 of the neutron star transient and the additional low-luminosity X-ray sources in NGC 6440 in more detail, we observed NGC 6440 for a third time using Chandra. Our observation was taken while this transient was in a quiescent state, and we compare this new Chandra observation with the previous Chandra observation of the transient in a quiescent state by in 't Zand et al. (2001) and Pooley et al. (2002) to study potential variability of the quiescent X-ray emission of the source. The other globular cluster low-luminosity sources within NGC 6440 will be discussed in a future paper.

\section{OBSERVATIONS AND ANALYSIS}

On 2003 June 26 we observed NGC 6440 with Chandra for 24 ks using only the S3 chip of the ACIS-S detector. In Figure 1 we show the RXTE ASM light curve of the transient since 1996 January. In this figure we indicate when the Chandra observations were performed. It can be seen that CX1 was not in a bright outburst phase during the 2000 July 4 observation and our 2003 June 26 observation (as we show below, CX1 was in a quiescent state during both observations). The location of the 1998 August outburst is also marked. This outburst is barely detected with the RXTE ASM, and in this figure the data are binned over 7 days, so only one bin is slightly above the noise (see also in 't Zand et al. 1999). Since 1999 February, the Proportional Counter Array (PCA) on board RXTE has been monitoring the Galactic center region, which includes NGC 6440, about twice a week (Swank \& Markwardt 2001). Since the start of this monitoring campaign, only the 2001 outburst has been detected (C. B. Markwardt 2004, private communication). The PCA is an order of magnitude more sensitive than the ASM, so weak outbursts that might have been missed by the ASM should have been detected by the PCA. However, we cannot exclude that before 1999 February, weak outbursts with X-ray fluxes below the ASM detection threshold may have gone undetected.

Data reduction and analysis of the 2003 June 26 and 2000 July 4 observations were performed using the CIAO version 3.0.2 software package provided by the Chandra X-ray Center and following the threads listed on the CIAO Web site. ${ }^{5}$ Background flares were searched for, but none were found, so we used all available data in our analysis.

\subsection{Image Analysis}

A color image of the cluster was produced from the 2003 June 26 observation (hereafter observation 2; see Fig. 2, right panel), and a similar image was produced from the 2000 July 4 observation for comparison (observation 1; see Fig. 2, left panel). It can be seen that during both of these observations, the

${ }^{5}$ Available at http://cxc.harvard.edu/ciao/.

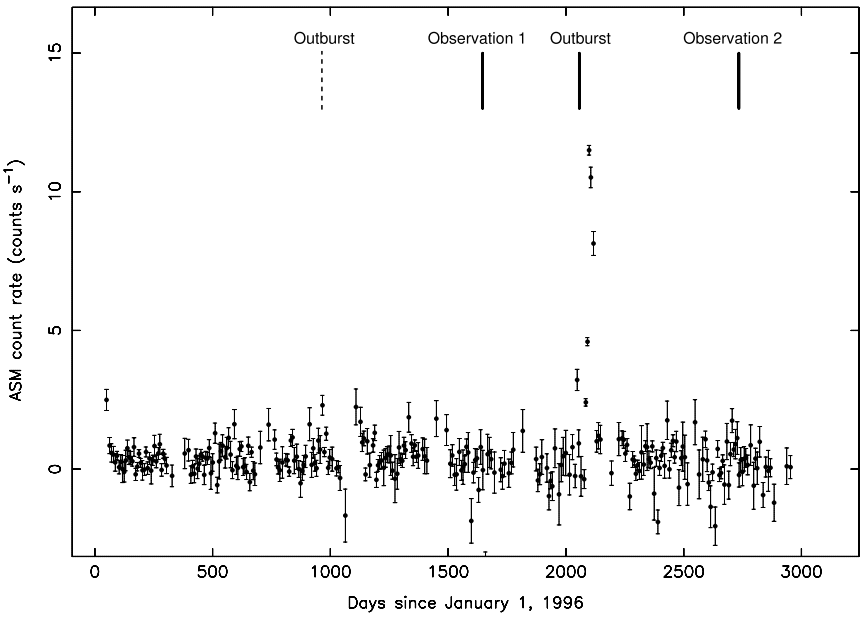

FIG. 1.- RXTE ASM light curve of the transient in NGC 6440. Each point is averaged over 7 days. The dates of the three Chandra observations are marked with solid lines. It can be seen that both observation 1 and observation 2 were taken while the neutron star X-ray transient in NGC 6440 was in a quiescent state. The dashed line indicates the 1998 August outburst.

source was in a quiescent state (see in 't Zand et al. [2001] for a comparison of observation 1 with the 2001 August Chandra observation of the source in outburst). In this figure, the red color is for the $0.3-1.5 \mathrm{keV}$ energy range, green is for $1.5-$ $2.5 \mathrm{keV}$, and blue is for $2.5-8.0 \mathrm{keV}$. When creating these images, we removed the pixel randomization that is added in the standard data processing; this slightly enhances the spatial resolution of the images. We used wavdetect to detect the point sources in the cluster and determine their positions. To determine that we had correctly identified CX1 in the new observation, we calculated the coordinate offset between the two observations of the five brightest sources detected (CX2-CX6, as named in Pooley et al. 2002). The mean offset in R.A. $=0$ "'39 and in decl. $=-0.07$, with standard deviations of these offsets being 0 ". 11 in R.A. and 0 ". 13 in decl. The offset of CX1 between the two observations was 0 ".41 in R.A. and -0 ".04 in decl. These offsets are well within $1 \sigma$ of the averaged offsets measured for the other sources; hence we conclude that we have detected the same source in both observations.

\subsection{Spectral Analysis}

Figure 2 shows a change in color of the source between the two observations. The more yellowish X-ray colors of the second observation suggest that the spectrum is softer in the second observation. Since the launch of Chandra there has been continuous degradation of the ACIS quantum efficiency, ${ }^{6}$ which is most severe at lower energies. The effect of this degradation would be to make the second observation harder, and hence this actually strengthens our impression, based on a comparison of the two images, that the spectrum of the second observation is softer.

We extracted the count rates using a circle of radius 1.5 centered on the source position as the source extraction region and an annulus from $17^{\prime \prime}$ to $28^{\prime \prime}$ centered on the cluster center as the background region. No sources were detected in this annulus. For the source CX1, we detected 247 photons for observation 1 (for the photon energy range $0.3-10 \mathrm{keV}){ }^{7}$ with

\footnotetext{
6 See http://cxc.harvard.edu/cal/Acis/Cal_prods/qeDeg/.

7 A total number of 251 photons are detected for observation 1 in the full energy range of Chandra, as previously found in in 't Zand et al. (2001).
} 

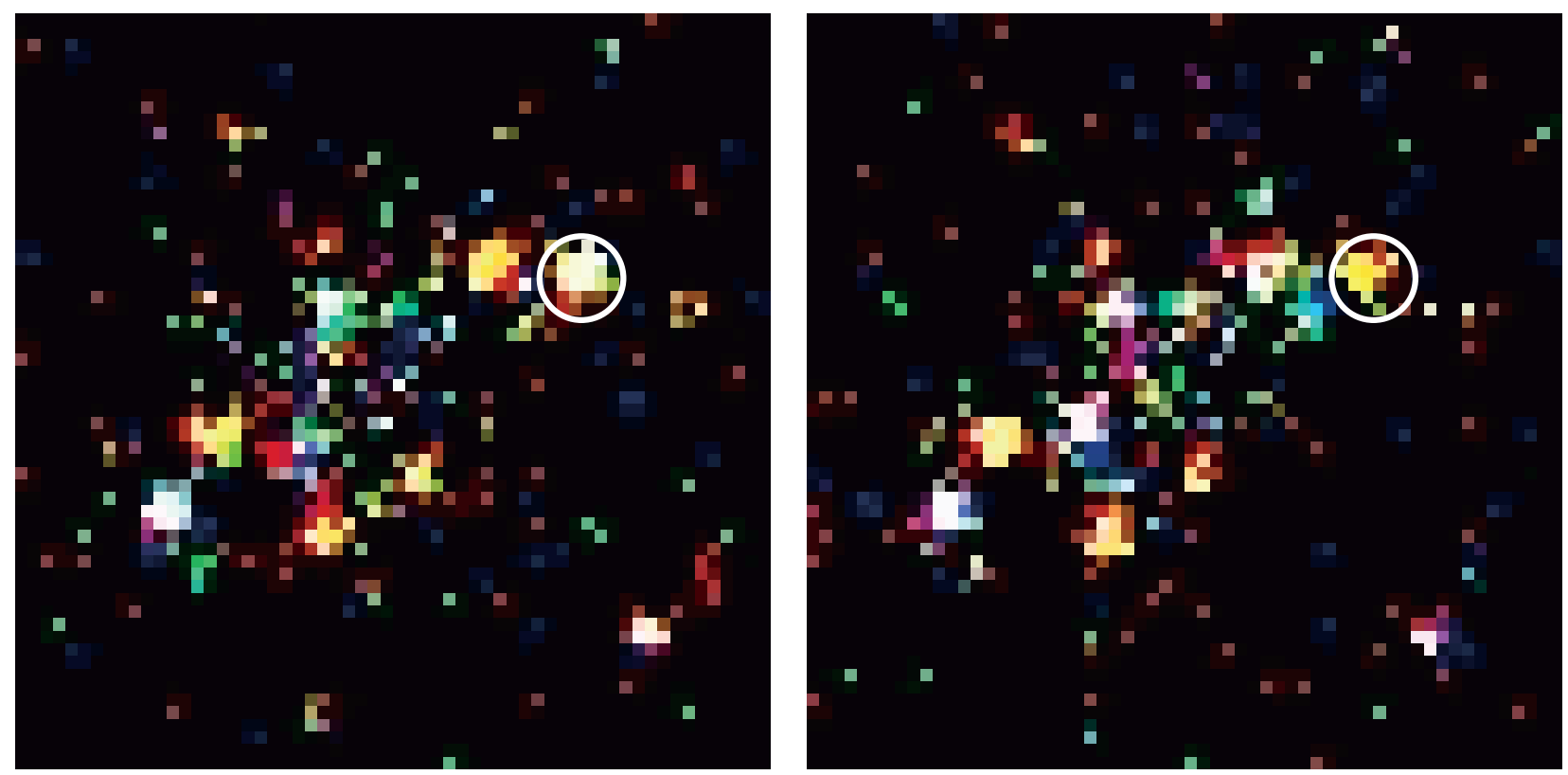

FIG. 2.- Images of the globular cluster NGC 6440. The left panel shows the data obtained during the 2000 July 4 Chandra observation (observation 1 ), and the

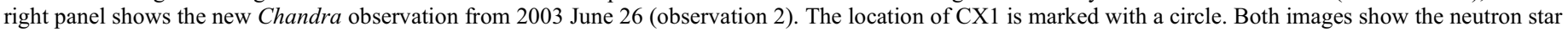

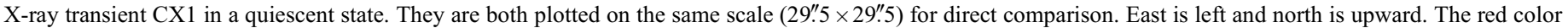
is for the $0.3-1.5 \mathrm{keV}$ energy range, green is for $1.5-2.5 \mathrm{keV}$, and blue is for $2.5-8.0 \mathrm{keV}$.

0.7 background photons, giving a net count rate of $0.0106 \pm$ 0.0007 counts $\mathrm{s}^{-1}$, compared to 108 photons for observation 2 (also for the photon energy range $0.3-10 \mathrm{keV}$ ), 0.6 background photons, and a net count rate of $0.0045 \pm 0.0004$ counts $\mathrm{s}^{-1}$. Clearly, we see a difference in the net count rates between the two observations, strongly suggesting that the source was variable between the two quiescent observations. To further investigate the spectral and count rate variations we observed, we extracted the source spectra using the randomized data and the same extraction regions as above by using the CIAO tool psextract. This also creates the response matrices and ancillary response files and automatically corrects the latter for the degradation of the low-energy quantum efficiency of the $\mathrm{CCD}$, as mentioned above. The spectrum was grouped into bins of 10 counts. More than $15-20$ counts bin ${ }^{-1}$ are formally required to use the $\chi^{2}$ statistic, but we decided on this number because the second observation had few net counts. We checked our results using the Cash statistics (Cash 1979) and found the results to be consistent with the $\chi^{2}$ method (within the $90 \%$ confidence levels); we only present results obtained using $\chi^{2}$ statistics. In our spectral analysis we use the neutron star hydrogen atmosphere (NSA) model for weakly magnetized neutron stars (Zavlin et al. 1996). Various other models, including blackbody and disk blackbody models, fit the data satisfactorily, but the NSA model is the most commonly used and currently accepted to be a better physical description of the emission originating from neutron star surfaces than other models (see, e.g., Rutledge et al. [1999, 2000] for an in-depth discussion).

\subsubsection{Individual Spectral Fits}

Initially, we fitted the two spectra separately using XSPEC version 11.2.0 (Arnaud 1996). For observation 1, the model used consists of an absorbed neutron star atmosphere model plus a power law. The inclusion of a power law to account for the hardest photons significantly improves the fit. For observation 2 , however, the inclusion of a power law does not improve the fit, and, in fact, when a power law is included, XSPEC forces the value of the power-law index to a high, unphysical value. For example, when assuming a distance to the source of $d=8.1,8.5$, or $8.9 \mathrm{kpc}$, the best-fitting power-law index is 9.5 in each case. An absorbed NSA model excluding a power law is therefore used when fitting the spectrum of observation 2 .

The normalization in the NSA model (Zavlin et al. 1996) is given as $1 / d^{2}$, where $d$ is the distance in parsecs. When leaving the normalization of the NSA model free, this parameter could not be constrained well, with values between $9.2 \times 10^{-7}$ and $1.2 \times 10^{-10}$ and a best-fit value of $5.0 \times 10^{-9}$. This corresponds to a distance range of $1-93 \mathrm{kpc}$ with a best-fit value of $14 \mathrm{kpc}$. Leaving the NSA normalization free leads to large uncertainties in the other parameters, too. The best-known distance to NGC 6440 from optical observations is well defined as being $8.5 \pm 0.4 \mathrm{kpc}$ (Ortolani et al. 1994), which is consistent with our range of distances. By fixing the NSA normalization, the uncertainties in the other parameters were reduced. We set the NSA normalization using $d=8.1,8.5$, and $8.9 \mathrm{kpc}$, fully covering the allowed distance range from the optical observations. A "canonical" neutron star with a mass of $1.4 M_{\odot}$ and a radius of $10 \mathrm{~km}$ was also assumed. Therefore, the only allowed free parameter in the NSA model was the temperature. When fitting to the individual observations, the column density and the power-law spectral index and normalization were free parameters. The results of the spectral fits to the individual observations are shown in Tables 1 and 2. From these results it can be seen that the different NSA normalization values do not greatly affect the fit parameters. The flux of the source is seen to change between the two observations. The model was extrapolated to the energy range $0.01-100 \mathrm{keV}$ to give an estimate of the bolometric flux. Between the two observations the 0.5$10 \mathrm{keV}$ flux decreases by a factor of $\sim 2$. To determine the errors in the fluxes, each free-fit parameter was fixed to its minimum or maximum value in turn, and only one at a time, with the exception of the power-law component. This component was fixed to its best-fitting value, as it is highly unconstrained and if left free gives unreasonably large errors (e.g., a factor of 
TABLE 1

Spectral Results for Observation 1

\begin{tabular}{|c|c|c|c|}
\hline \multirow[b]{2}{*}{ Model Parameter } & \multicolumn{3}{|c|}{ Assumed Distance to NGC 6440} \\
\hline & $8.1 \mathrm{kpc}$ & $8.5 \mathrm{kpc}$ & $8.9 \mathrm{kpc}$ \\
\hline$N_{\mathrm{H}}\left(10^{22} \mathrm{~cm}^{-2}\right) .$. & $0.7 \pm 0.1$ & $0.7 \pm 0.1$ & $0.7 \pm 0.1$ \\
\hline 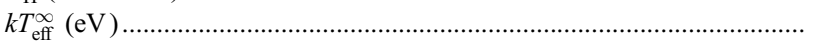 & $88 \pm 5$ & $91 \pm 5$ & $92 \pm 5$ \\
\hline 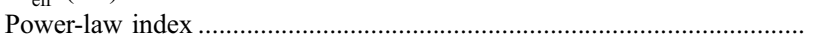 & $2.5 \pm 1.0$ & $2.4 \pm 1.0$ & $2.4 \pm 1.0$ \\
\hline 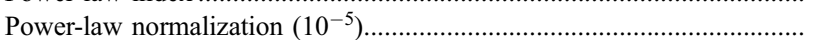 & $2.2_{-14}^{+3.5}$ & $2.0_{-13}^{+3.4}$ & $1.9_{-13}^{+3.3}$ \\
\hline 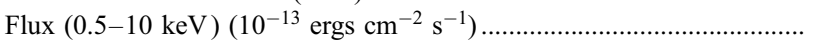 & $1.9 \pm 0.4$ & $1.8 \pm 0.4$ & $1.8 \pm 0.4$ \\
\hline 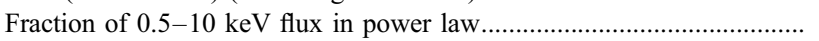 & $0.41 \pm 0.2$ & $0.40 \pm 0.2$ & $0.40 \pm 0.2$ \\
\hline NSA contribution to the bolometric flux $\left(10^{-13} \mathrm{ergs}^{-2} \mathrm{~cm}^{-1}\right) \ldots \ldots \ldots \ldots . . . . .$. & $1.7 \pm 0.4$ & $1.7 \pm 0.4$ & $1.6 \pm 0.4$ \\
\hline$\chi_{\nu}^{2}$ & 0.74 & 0.73 & 0.73 \\
\hline
\end{tabular}

NotE.-The errors represent $90 \%$ confidence levels. For the NSA model, we used a neutron star mass of $1.4 M_{\odot}$, a radius of $10 \mathrm{~km}$, and the neutron star hydrogen atmosphere model for weakly magnetized neutron stars of Zavlin et al. (1996).

approximately $10^{2}$ ). The model was then refitted to the data and new flux values calculated. Once this was done for every free parameter, the total flux range was used to give the flux errors.

\subsubsection{Combined Spectral Fits}

To further investigate the cause behind the variation in flux, we fit the two observations simultaneously (Table 3 ). The spectra and fitted models can be seen in Figure 3. As we expect the column density to be very similar for each observation, it was decided to tie this parameter between observations. Again, a canonical neutron star was used, and only the temperature in the NSA model was left as a free parameter. The power-law spectral index and normalization for the first observation were allowed to be free, whereas the normalization was initially fixed to zero for the second observation. After the model had been fit to the data, the power-law spectral index of the second observation was fixed to the value obtained for the first observation, and the $90 \%$ confidence limit on the normalization was determined. This gives us an indication of the upper limit of the power-law normalization for the second observation, assuming that the power-law spectral index was the same for both observations. In this case, taking the distance to the source to be $8.5 \mathrm{kpc}$, we get an upper limit on the power-law normalization of $1.7 \times 10^{-5}$, with the power-law index being 2.5 . This gives a corresponding upper limit on the unabsorbed $0.5-10 \mathrm{keV}$ flux of $1.0 \times 10^{-13} \mathrm{ergs} \mathrm{cm}^{-2} \mathrm{~s}^{-1}$ and hence the maximum contribution of the power-law component to the $0.5-10 \mathrm{keV}$ flux of $10 \%$. Similar upper limits were determined when taking $d=$ 8.1 and $8.9 \mathrm{kpc}$ (see Table 3). Power-law indices that are lower than our observed value for the first observation have been measured in other systems (e.g., Rutledge et al. 1999), and it is possible that the power-law index could have changed between the observations. Therefore, to investigate whether the powerlaw index or normalization is variable between the two observations, the upper limits were also determined when setting the power-law spectral index of the second observation to 0.5 and 1.0. The corresponding upper limits in the power-law normalization were $2.9 \times 10^{-6}$ and $4.3 \times 10^{-6}$, respectively, when taking the distance of the source to be $8.5 \mathrm{kpc}$. This gives unabsorbed $0.5-10 \mathrm{keV}$ fluxes of $1.6 \times 10^{-13}$ and $1.3 \times 10^{-13} \mathrm{ergs}$ $\mathrm{cm}^{-2} \mathrm{~s}^{-1}$ and the maximum contributions of the power-law component to the $0.5-10 \mathrm{keV}$ flux of $44 \%$ and $31 \%$, respectively. As the upper limit to the power-law normalization for observation 2 is strongest when the power law has a spectral index equal to that of the first observation, it is possible that the power-law index remains unchanged between the observations, and it is the normalization that has changed, although we cannot be conclusive.

Assuming that the distance to the source is $8.5 \mathrm{kpc}$, we found an unabsorbed $0.5-10 \mathrm{keV}$ flux of $(1.9 \pm 0.3) \times 10^{-13} \mathrm{ergs}$ $\mathrm{cm}^{-2} \mathrm{~s}^{-1}$ in observation 1 and $(0.9 \pm 0.2) \times 10^{-13} \mathrm{ergs} \mathrm{cm}^{-2}$ $\mathrm{s}^{-1}$ in observation 2 . Our results show that the unabsorbed $0.5-$ $10 \mathrm{keV}$ flux reduces by a factor of $2.1 \pm 0.6$ between the first and second observations. This confirms our conclusion ( $(2.2)$ that the neutron star X-ray transient in NGC 6440 exhibits variability in quiescence. The contribution to the bolometric flux from the NSA component was found to be $(1.6 \pm 0.4) \times$ $10^{-13} \mathrm{ergs} \mathrm{cm}^{-2} \mathrm{~s}^{-1}$ in observation 1 , whereas all the bolometric flux comes from the NSA component in observation 2 and is found to be $(1.4 \pm 0.2) \times 10^{-13} \mathrm{ergs} \mathrm{cm}^{-2} \mathrm{~s}^{-1}$. So, the NSA component of the bolometric flux is seen to be consistent between the two observations. The corresponding effective temperatures

TABLE 2

Spectral Results for Observation 2

\begin{tabular}{|c|c|c|c|}
\hline \multirow[b]{2}{*}{ Model Parameter } & \multicolumn{3}{|c|}{ Assumed Distance to NGC 6440} \\
\hline & $8.1 \mathrm{kpc}$ & $8.5 \mathrm{kpc}$ & $8.9 \mathrm{kpc}$ \\
\hline 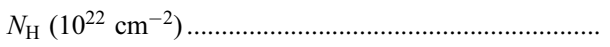 & $0.7 \pm 0.2$ & $0.7 \pm 0.2$ & $0.7 \pm 0.2$ \\
\hline$k T_{\mathrm{eff}}^{\infty}(\mathrm{eV})$ & $85 \pm 5$ & $87 \pm 5$ & $88 \pm 5$ \\
\hline Flux $(0.5-10 \mathrm{keV})\left(10^{-13}\right.$ ergs $\left.\mathrm{cm}^{-2} \mathrm{~s}^{-1}\right) \ldots \ldots \ldots \ldots \ldots \ldots \ldots \ldots$ & $0.9 \pm 0.3$ & $0.9 \pm 0.2$ & $0.9 \pm 0.2$ \\
\hline 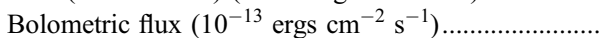 & $1.5 \pm 0.3$ & $1.4 \pm 0.3$ & $1.4 \pm 0.3$ \\
\hline 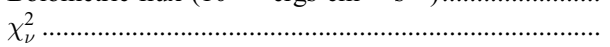 & 0.66 & 0.66 & 0.65 \\
\hline
\end{tabular}

NotE.-The errors represent $90 \%$ confidence levels. For the NSA model, we used a neutron star mass of $1.4 M_{\odot}$, a radius of $10 \mathrm{~km}$, and the neutron star hydrogen atmosphere model for weakly magnetized neutron stars of Zavlin et al. (1996). 
TABLE 3

Spectral Results when Simultaneously Fitting to Observations 1 and 2

\begin{tabular}{|c|c|c|c|}
\hline \multirow[b]{2}{*}{ Model Parameter } & \multicolumn{3}{|c|}{ Assumed Distance to NGC 6440} \\
\hline & $8.1 \mathrm{kpc}$ & $8.5 \mathrm{kpc}$ & $8.9 \mathrm{kpc}$ \\
\hline \multicolumn{4}{|c|}{ Parameters for Observation 1} \\
\hline 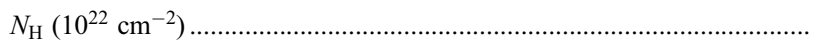 & $0.7 \pm 0.1$ & $0.7 \pm 0.1$ & $0.7 \pm 0.1$ \\
\hline$k T_{\text {eff }}^{\infty}(\mathrm{eV})$ & $87 \pm 5$ & $89 \pm 4$ & $92 \pm 5$ \\
\hline Power-law index & $2.6 \pm 0.8$ & $2.5 \pm 0.8$ & $2.4 \pm 0.9$ \\
\hline 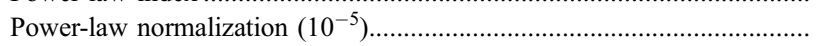 & $2.7_{-1.5}^{+2.7}$ & $2.3_{-1.3}^{+2.5}$ & $2.0_{-1.2}^{+2.3}$ \\
\hline 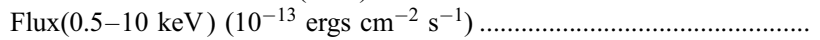 & $1.9 \pm 0.3$ & $1.9 \pm 0.3$ & $1.8 \pm 0.3$ \\
\hline 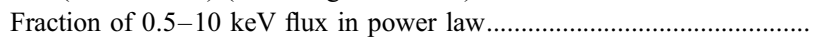 & $0.46 \pm 0.2$ & $0.43 \pm 0.2$ & $0.40 \pm 0.2$ \\
\hline NSA contribution to the bolometric flux $\left(10^{-13} \mathrm{ergs}^{-2} \mathrm{~cm}^{-1}\right) \ldots \ldots \ldots \ldots$ & $1.6 \pm 0.4$ & $1.6 \pm 0.4$ & $1.6 \pm 0.3$ \\
\hline \multicolumn{4}{|c|}{ Parameters for Observation 2} \\
\hline$k T_{\text {eff }}^{\infty}(\mathrm{eV})$ & $85 \pm 4$ & $86 \pm 4$ & $88 \pm 4$ \\
\hline 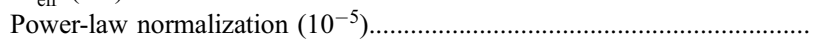 & $<2.0$ & $<1.7$ & $<1.5$ \\
\hline 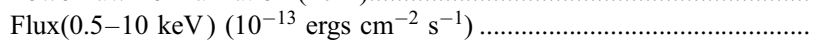 & $0.9 \pm 0.2$ & $0.9 \pm 0.2$ & $0.9 \pm 0.2$ \\
\hline 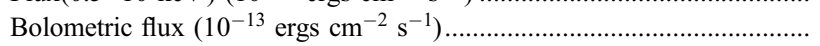 & $1.5 \pm 0.2$ & $1.4 \pm 0.2$ & $1.4 \pm 0.2$ \\
\hline$\chi_{\nu}^{2}$ & 0.69 & 0.68 & 0.68 \\
\hline
\end{tabular}

Note.-The errors represent $90 \%$ confidence levels. For the NSA model, we used a neutron star mass of $1.4 M_{\odot}$, a radius of $10 \mathrm{~km}$, and the neutron star hydrogen atmosphere model for weakly magnetized neutron stars of Zavlin et al. (1996).

of the thermal component for the two observations are seen to remain constant to within the $68.3 \%$ confidence level. This is illustrated in Figure 4, in which the line of equal temperature between the observations goes within this $68.3 \%$ confidence contour.

\section{DISCUSSION}

We have presented a new Chandra observation of the globular cluster NGC 6440 during a time when the neutron star $\mathrm{X}$-ray transient CX1 was in a quiescent state and compared this observation with a previous Chandra observation also taken while CX1 was in a quiescent state. Both spectra have been acceptably fitted with NSA models using a "canonical" neutron star at the distance of NGC 6440 combined with Galactic

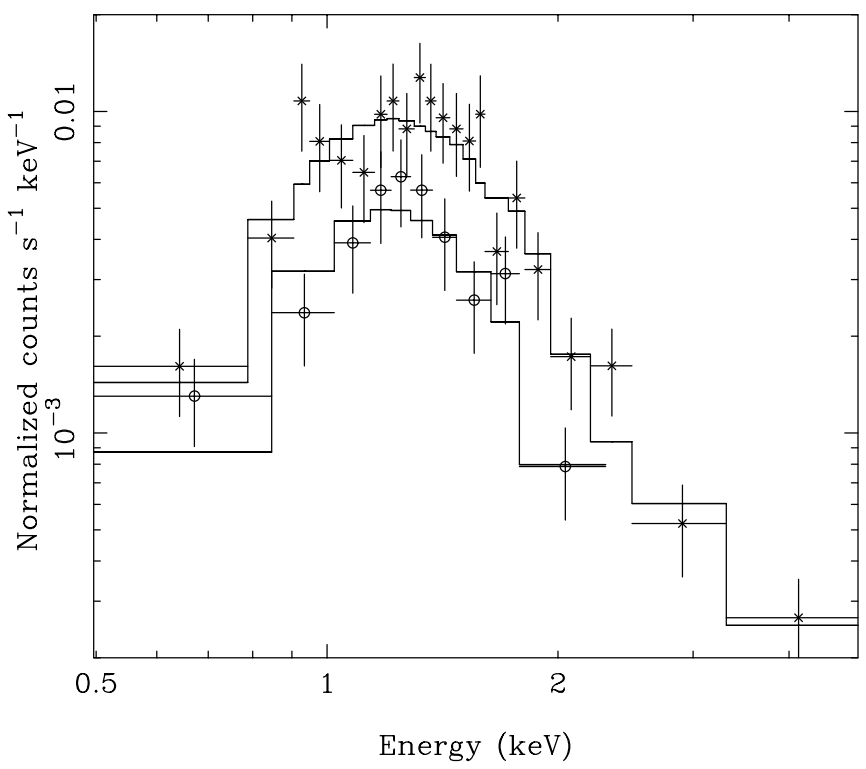

FIG. 3.-X-ray spectra of the X-ray transient neutron star in NGC 6440 during observation 1 (crosses) and observation 2 (circles). The solid lines through the data points are the best-fitting models to the data. [See the electronic edition of the Journal for a color version of this figure.] absorption. In observation 1 the addition of a power-law component improves the fit at higher energies, as has previously been found for other quiescent neutron stars (e.g., Aql X-1 [Rutledge et al. 2001a, 2002; Campana et al. 1998b] and Cen X-4 [Rutledge et al. 2001b; Campana et al. 2004]), but the addition of such a term to the second observation does not improve the fit. We have shown that the $0.5-10 \mathrm{keV}$ flux is seen to decrease by a factor of $\sim 2$ between these two observations, and that the neutron star atmosphere component to the bolometric flux is seen to be consistent. Our results for observation 1 are found to be consistent with the previous analysis by in 't Zand et al. (2001).

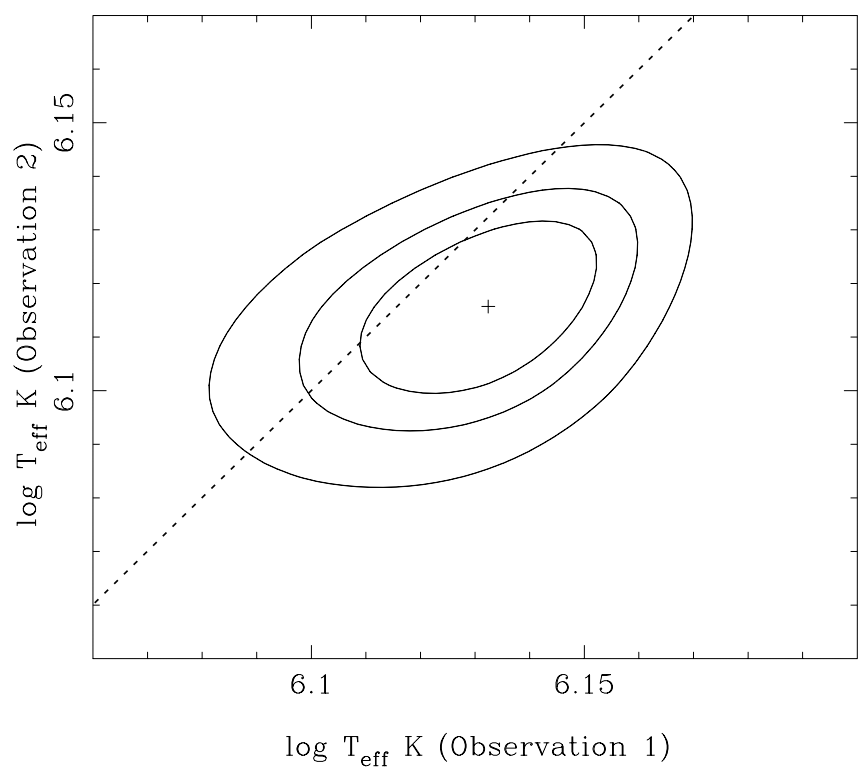

FIG. 4.-Comparison of the effective temperature (for an observer on the surface of the neutron star) between observations 1 and 2 when taking the distance to the source to be $8.5 \mathrm{kpc}$. The contours mark the $68.3 \%, 90 \%$, and $99 \%$ confidence levels. The dashed line indicates where the temperature of observation 1 equals the temperature of observation 2 . The contours were determined with the power law fixed to the best-fitting value. [See the electronic edition of the Journal for a color version of this figure.] 
Other quiescent neutron stars that have been observed to be variable during quiescence include Aql X-1, Cen X-4, MXB 1659-29, and KS 1731-260. Rutledge et al. (2001a, 2002) ascribe the variability in Aql X-1 to a change in the neutron star effective temperature caused by variable residual accretion onto the neutron star, whereas Campana \& Stella (2003) prefer to explain the variability as due to correlated variations of the power-law component and the local column density, supporting the idea of shock emission. The variability in Cen X-4 has been attributed to a changing power-law component (Rutledge et al. 2001b), a variable local column density combined with variation in either the soft or hard spectral component, or both (Campana et al. 2004). Long accretion events in MXB 165929 and KS 1731-260 may have heated the crust of the neutron stars in these systems considerably out of thermal equilibrium with the cores, and the quiescent variability can be explained by the cooling of the crusts toward renewed equilibrium with the cores (Wijnands et al. 2002, 2004). However, the outbursts of the transient in NGC 6440 are short (compared to the outbursts of MXB 1659-29 and KS 1731-260), during which the neutron star crust is only slightly heated, and soon (within weeks; e.g., Brown et al. 1998) after the end of the outbursts, the crust is in thermal equilibrium with the core. Since our quiescent observations were both taken $\sim 2 \mathrm{yr}$ after the end of the outbursts, we expect that the crust has already reached equilibrium with the core, and therefore a cooling crust probably cannot explain the observed variability.

From our spectral analysis of CX1, the significant difference between the observations to note is the undetectability of a power-law component in the second observation. Although a hard power-law component is seen in observation 1 , it is not detected in observation 2. Both observations were of the same exposure and so will be equally sensitive to the hard tail. For the photon energy range $3-10 \mathrm{keV}, 15.7$ net counts were detected from the source in the first observation ( 0.3 background counts), whereas no counts were detected in this energy range in the second observation. Clearly, there is a definite change in the power-law component between these observations. However, it is unclear from these results whether this is due to a variable spectral index, normalization, or both. This change in property, or properties, of the power-law component likely accounts for almost all of the change in luminosity. The unchanging neutron star effective temperature between the observations supports the idea that the thermal quiescent luminosity is set by deep crustal heating (Brown et al. 1998).

We have clearly shown that the neutron star X-ray transient in NGC 6440 was variable between two quiescent observations. This variability is attributable to a change in the power-law component, while the thermal component is consistent with remaining constant. This is similar to the results found for Aql X-1 (Campana \& Stella 2003) and Cen X-4 (Campana et al. 2004), in which the thermal component from the neutron star is consistent with remaining constant, and the power law is seen to be variable. These observations all indicate that there is a stable thermal flux coming from the neutron star surface, likely set by deep crustal heating, and a variable flux at higher energies. Although the nature of the power-law component is uncertain, one proposal is that it could be due to matter interacting with the magnetic field of the neutron star in some way (e.g., Campana et al. 1998a; Campana \& Stella 2000). In particular, Campana \& Stella (2003) and Campana et al. (2004) explain the variable power-law flux they observed as emission at the shock between a pulsar wind and a variable amount of inflowing matter from the companion star. They base this on the fact that they found evidence that the spectral variability in Aql X-1 and Cen X-4 could be interpreted as correlated variations of the power-law component and the local column density. Unfortunately, we were unable to determine if a similar correlation in CX1 was underlying the spectral variability we found in this source, as we cannot fit a power law to both observations. However, some variation in accretion rate onto the magnetosphere or variation in the interaction of the pulsar wind with the matter still outflowing from the companion star could, in principle, explain the variability observed in CX1, with less matter interacting with the neutron star magnetic field during observation 2 than during observation 1 . If there is residual accretion occurring, it is unlikely to accrete down to the surface, since that would produce thermal-like emission (Zampieri et al. 1995), due to the heating of the surface, over the thermal flux expected from a cooling neutron star.

The transient in NGC 6440 was seen to be in a quiescent state from the outburst in 1971 until the 1998 outburst and then until the 2001 outburst. We can use this information to predict the thermal flux during quiescence. From the time averaged accretion flux, $\left\langle F_{\text {acc }}\right\rangle$, the expected thermal flux in quiescence, $F_{q}$, can be predicted using the Brown et al. (1998) model and assuming standard core cooling: $F_{q}=\left\langle F_{\text {acc }}\right\rangle / 135$ (Brown et al. 1998; Wijnands et al. 2001; Rutledge et al. 2002). From this, it can be derived (Wijnands et al. 2001) that $F_{q} \sim\left[t_{o} /\left(t_{o}+t_{q}\right)\right]$ $\left(\left\langle F_{o}\right\rangle / 135\right)$, where $\left\langle F_{o}\right\rangle$ is the time-averaged flux during outburst, $t_{o}$ is the time the source was in outburst, and $t_{q}$ is the averaged time the source is in quiescence. The time-averaged flux during outburst for CX1 can be estimated from the RXTE ASM light curve (see Fig. 1). To convert the ASM count rate to a flux, we use WebPIMMS, modeling the outburst spectrum as a power law with index 1.7 and $N_{\mathrm{H}}=1.2 \times 10^{22} \mathrm{~cm}^{-2}$ (valid for the energy range $1-40 \mathrm{keV}$; in 't Zand et al. 1999). This gives the time-averaged flux during outburst as $\left\langle F_{o}\right\rangle=5.79 \times$ $10^{-9} \mathrm{ergs} \mathrm{cm}^{-2} \mathrm{~s}^{-1}$ in the energy range $1-40 \mathrm{keV}$. However, the bolometric flux could be a factor of a few higher than this depending on the correct spectral model and energy range. Assuming that no other outbursts were detected since the 1971 outburst, we get the total time in quiescence up until the 2001 outburst as $t_{q}=10,973$ days, estimating the total outburst time $t_{o} \sim 43$ days (from the ASM light curve). The predicted qui-

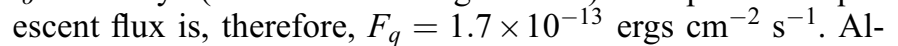
though the bolometric correction could make this predicted flux a factor of a few higher, the uncertainties in our assumptions are likely to be large, making this predicted flux in good agreement with the observed quiescent flux of the NSA component that we found from the Chandra observations (Tables 1-3). This shows that the standard cooling model can account for the observed behavior of CX1. Other quiescent neutron stars cannot easily be explained by the standard cooling model and require enhanced core cooling: for example, KS 1731-260 (Wijnands et al. 2001) and Cen X-4 (Rutledge et al. 2001b), in which case the neutron stars in these systems could be more massive $\left(>1.7 M_{\odot}\right)$ than those in prototypical neutron star transients (e.g., Colpi et al. 2001). Further monitoring of this and other neutron star transients in a quiescent state will better constrain the quiescent properties and help determine the cause of the observed variability.

E. M. C. acknowledges the support of a Particle Physics and Astronomy Research Council (PPARC) Studentship at the University of St. Andrews. 
Arnaud, K. A. 1996, in ASP Conf. Ser. 101, Astronomical Data Analysis Software and Systems V, ed. G. H. Jacoby \& J. Barnes (San Francisco: ASP), 17 Brown, E. F., Bildsten, L., \& Rutledge, R. E. 1998, ApJ, 504, L95

Campana, S., Colpi, M., Mereghetti, S., Stella, L., \& Tavani, M. 1998a, A\&A Rev., 8, 279

Campana, S., Israel, G. L., Stella, L., Gastaldello, F., \& Mereghetti, S. 2004, ApJ, 601, 474

Campana, S., \& Stella, L. 2000, ApJ, 541, 849 . 2003, ApJ, 597, 474

Campana, S., Stella, L., Mereghetti, S., Colpi, M., Tavani, M., Ricci, D., Fiume, D. D., \& Belloni, T. 1998b, ApJ, 499, L65

Cash, W. 1979, ApJ, 228, 939

Colpi, M., Geppert, U., Page, D., \& Possenti, A. 2001, ApJ, 548, L175

Forman, W., Jones, C., \& Tananbaum, H. 1976, ApJ, 207, L25

in 't Zand, J., Heise, J., Bazzano, A., Ubertini, P., Smith, M. J. S., Muller, J. M., \& Torroni, V. 1998, IAU Circ., 6997, 3

in 't Zand, J. J. M., van Kerkwijk, M. H., Pooley, D., Verbunt, F., Wijnands, R., \& Lewin, W. H. G. 2001, ApJ, 563, L41

in 't Zand, J. J. M., et al. 1999, A\&A, 345, 100

Markert, T. H., Backman, D. E., Canizares, C. R., Clark, G. W., \& Levine, A. M. 1975, Nature, 257, 32

\section{EFERENCES}

Menou, K., Hameury, J., \& Stehle, R. 1999, MNRAS, 305, 79

Menou, K., \& McClintock, J. E. 2001, ApJ, 557, 304

Ortolani, S., Barbuy, B., \& Bica, E. 1994, A\&AS, 108, 653

Pooley, D., et al. 2002, ApJ, 573, 184

Rutledge, R. E., Bildsten, L., Brown, E. F., Pavlov, G. G., \& Zavlin, V. E. 1999, ApJ, 514, 945

$$
\begin{aligned}
& 2000, \text { ApJ, 529, } 985 \\
& 2001 \mathrm{a}, \text { ApJ, 559, } 1054 \\
& 2001 \mathrm{~b}, \text { ApJ, 551, } 921 \\
& 2002, \text { ApJ, 577, } 346
\end{aligned}
$$

Swank, J., \& Markwardt, C. 2001, in ASP Conf. Ser. 251, New Century of X-ray Astronomy, ed. H. Inoue \& H. Kunieda (San Francisco: ASP), 94

Verbunt, F., van Kerkwijk, M. H., in’t Zand, J. J. M., \& Heise, J. 2000, A\&A, 359,960

Wijnands, R., Guainazzi, M., van der Klis, M., \& Méndez, M. 2002, ApJ, 573, L45

Wijnands, R., Homan, J., Miller, J. M., \& Lewin, W. H. G. 2004, ApJ, 606, L61 Wijnands, R., Miller, J. M, Markwardt, C., Lewin, W. H. G., \& van der Klis, M. 2001, ApJ, 560, L159

Zampieri, L., Turolla, R., Zane, S., \& Treves, A. 1995, ApJ, 439, 849

Zavlin, V. E., Pavlov, G. G., \& Shibanov, Y. A. 1996, A\&A, 315, 141 